\title{
Role of non-collective excitations in subbarrier fusion reactions
}

\author{
K. Hagino ${ }^{1}$, S. Yusa ${ }^{1}$, and N. Rowley ${ }^{2}$ \\ 1 Department of Physics, Tohoku University, Sendai 980-8578, Japan \\ 2 Institut de Physique Nucléaire, UMR 8608, CNRS-IN2P3 et Université de Paris Sud, 91406 Orsay Cedex, France
}

\begin{abstract}
We carry out a large scale coupled-channels calculations in order to discuss the effect of noncollective excitations on heavy-ion fusion and quasi-elastic scattering at subbarrier energies. To this end, we take into account all the states in ${ }^{208} \mathrm{~Pb}$ below $7 \mathrm{MeV}$, which were previously identified with a high resolution proton inelastic scattering measurement. Our calculations indicate that the energy dependence of the fusion and quasi-elastic cross sections are not altered much due to the non-collective coupling, although the peak structure in the barrier distributions is slightly smeared. We also show that our calculations well reproduce the energy dependence of the $Q$-value distribution for backward quasi-elastic scattering.
\end{abstract}

\section{Introduction}

The coupled-channels approach has been a standard tool to analyze heavy-ion reactions at energies close to the Coulomb barrier[1]. It has successfully accounted for the subbarrier enhancement of fusion cross sections, and at the same time, it has provided a natural explanation for the fusion barrier distribution through the eigen-channel representation[2,3].

Conventionally, in the coupled-channels framework, one takes into account those states which are strongly connected to the ground state. These are low-lying collective phonon states as well as the rotational states within the ground rotational band. Even though the coupling to the ground state is strong, giant resonances are usually not included since the excitation energy is rather large and thus the coupling simply leads to a potential renormalization [4]. Notice also that the giant resonances have a smooth mass number dependence, in contrast to the low-lying collective motions. Other excitations, that is, non-collective excitations have either been neglected completely or taken into account only through the imaginary part of the internuclear potential.

Recently, quasi-elastic scattering cross sections for ${ }^{20} \mathrm{Ne}$ $+{ }^{90,92} \mathrm{Zr}$ systems at backward angles have been measured, which show a considerable difference in the barrier distribution between the two systems [5]. That is, the barrier distribution with the ${ }^{92} \mathrm{Zr}$ target is much more smeared than that with ${ }^{90} \mathrm{Zr}$. The coupled-channels calculations with lowlying collective states both in the projectile and the target, on the other hand, predict a similar barrier distribution to each other for both the systems. This is due to the fact that the rotational excitations of ${ }^{20} \mathrm{Ne}$ play a predominant role in these systems. Since those coupled-channels calculations have already included the collective excitations in the ${ }^{90,92} \mathrm{Zr}$ nuclei, the experimental data strongly suggest that the difference in the barrier distribution for the two systems can be attributed to non-collective excitations in the target nuclei.

In this contribution, we will explore the role of noncollective excitations in heavy-ion fusion and quasi-elastic scattering at energies around the Coulomb barrier [6]. To this end, we will particularly analyse the ${ }^{16} \mathrm{O}+{ }^{208} \mathrm{~Pb}$ reaction. Notice that the non-collective states are weakly coupled to the ground state, but there are many - the level density increases exponentially as the excitation energy increases. By treating the non-collective excitations in ${ }^{208} \mathrm{~Pb}$ explicitly in the coupled-channels approach, we will investigate whether the non-collective excitations resolve recent challenges in the coupled-channels approach, such as the surface diffuseness anomaly in the inter-nuclear potential in the fusion channel [7], the steep fall-off of fusion cross sections at deep subbarrier energies [8-10], a large smoothing of quasi-elastic barrier distribution [11,5], and the energy dependence of the $Q$-value spectra for quasielastic back scattering[12,13].

\section{Non-collective excitations in ${ }^{16} \mathrm{O}+{ }^{208} \mathrm{~Pb}$ reaction}

For the ${ }^{208} \mathrm{~Pb}$ nucleus, proton inelastic scattering has been measured in high energy resolution [14-17]. For example, Ref. [14] has found 64 levels up to $7 \mathrm{MeV}$ besides the collective $3^{-}$state at $2.61 \mathrm{MeV}, 5^{-}$state at $3.709 \mathrm{MeV}$, and $2^{+}$state at $4.0855 \mathrm{MeV}$. From the fit of the angular distribution with distorted wave Born approximation (DWBA) calculations, the deformation parameter $\beta_{\lambda}$ for these states have also been obtained. This information has been used by Demetriou, Marcinkowski, and Hodgson to discuss a multistep direct reaction mechanism [18].

We solve the coupled-channels equations by including those non-collective states in ${ }^{208} \mathrm{~Pb}$ below $7.382 \mathrm{MeV}$, together with the collective phonon states in ${ }^{208} \mathrm{~Pb}$ and the 


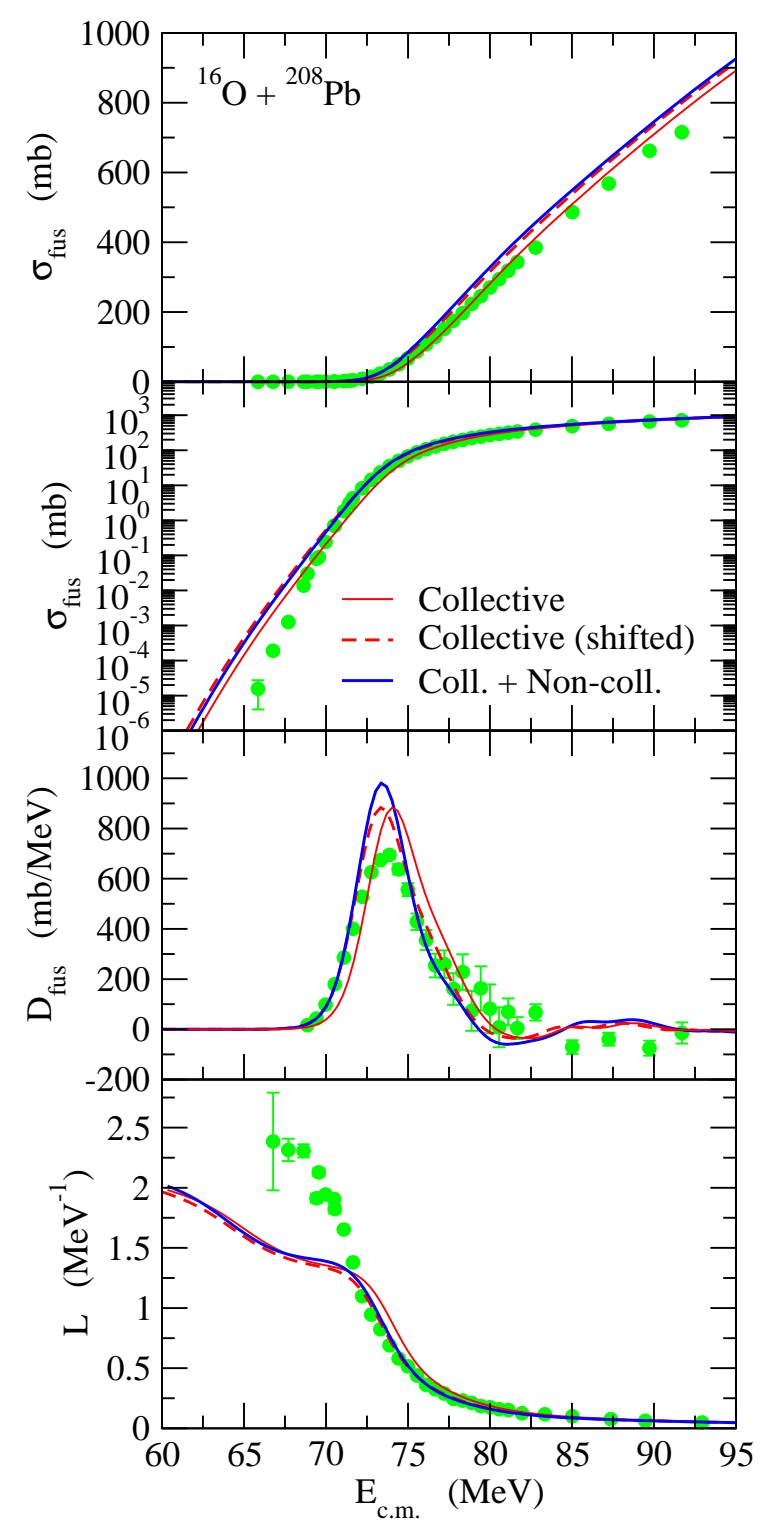

Fig. 1. Fusion cross sections for the ${ }^{16} \mathrm{O}+{ }^{208} \mathrm{~Pb}$ reaction. The first and the second panels show the cross sections in the linear and the logarithmic scales, respectively. The third and the fourth panels show the fusion barrier distribution, $D_{\text {fus }}=d^{2}\left(E \sigma_{\text {fus }}\right) / d E^{2}$, and the logarithmic slope, $L(E)=d\left[\ln \left(E \sigma_{\text {fus }}\right)\right] / d E$, respectively. The thin solid lines show the results of the coupled-channels calculations obtained only with the collective excitations, while the thick solid lines take into account also the non-collective excitations. The dashed lines are obtained by shifting in energy the thin solid lines. The experimental data are taken from Refs. [22,23].

collective $3^{-}$state in ${ }^{16} \mathrm{O}$ at $6.13 \mathrm{MeV}$. We also take into account the double octupole phonon excitations in ${ }^{208} \mathrm{~Pb}$ in the harmonic approximation. For the non-collective excitations, we assume a vibrational coupling between the ground state and the non-collective states, without taking into account the couplings among the non-collective states and the couplings between the collective and the non-collective states. For simplicity, we assume that the coupling form factors for the non-collective excitations are given by the

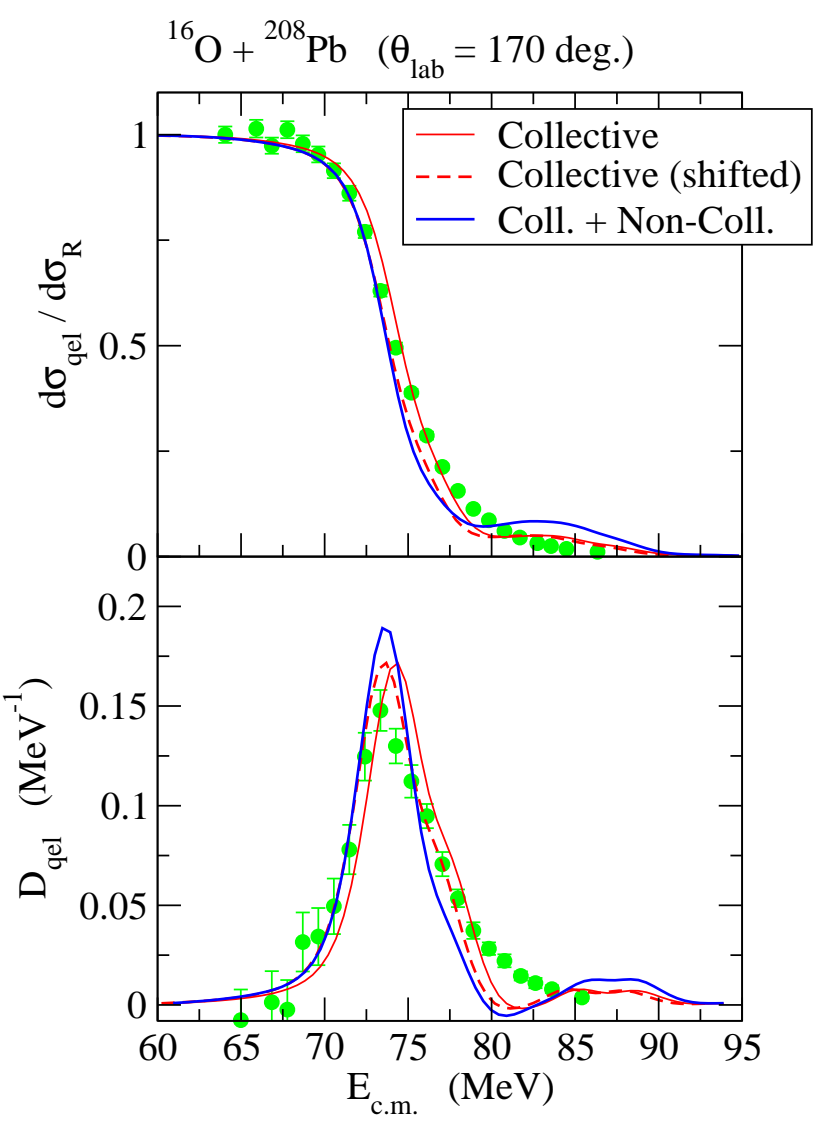

Fig. 2. The cross sections for the quasi-elastic ${ }^{16} \mathrm{O}+{ }^{208} \mathrm{~Pb}$ scattering at $\theta_{\text {lab }}=170^{\circ}$ and the quasi-elastic barrier distribution, $D_{\text {qel }}=$ $-d\left[d \sigma_{\mathrm{qel}} / d \sigma_{R}\right] / d E, \sigma_{R}$ being the Rutherford cross section. The meaning of each line is the same as in Fig. 1. The experimental data are taken from Ref. [24].

derivative of the internuclear potential (notice that we do not use the constant coupling approximation in contrast to Ref. [6]). Even though the radial dependence may differ for each non-collective state, we nevertheless introduce this approximation in order to be consistent with the DWBA analysis in Ref. [14]. We include the mutual excitations of the projectile and the target nuclei only for the octupole states in ${ }^{16} \mathrm{O}$ and ${ }^{208} \mathrm{~Pb}$. By introducing the isocentrifugal approximation [19], the total dimension of the coupledchannels equations is then reduced to 77 . For an internuclear potential, we use a Woods-Saxon potential that has the same geometry as the Akyüz-Winther potential [20]. We use the computer code CCFULL [1] to integrate the coupledchannels equations.

Figure 1 shows the fusion cross sections $\sigma_{\text {fus }}$ thus obtained. The barrier distribution, $D_{\text {fus }}=d^{2}\left(E \sigma_{\text {fus }}\right) / d E^{2}$, and the logarithmic derivative, $L(E)=d\left[\ln \left(E \sigma_{\text {fus }}\right)\right] / d E$, are also shown. The thin solid line is obtained by including only the collective states in the coupled-channels equations, while the thick solid line is obtained by including both the collective and the non-collective states. As one sees, the primary effect of the non-collective excitations is to shift the excitation functions in energy. This is understandable as the non-collective states have relatively high 


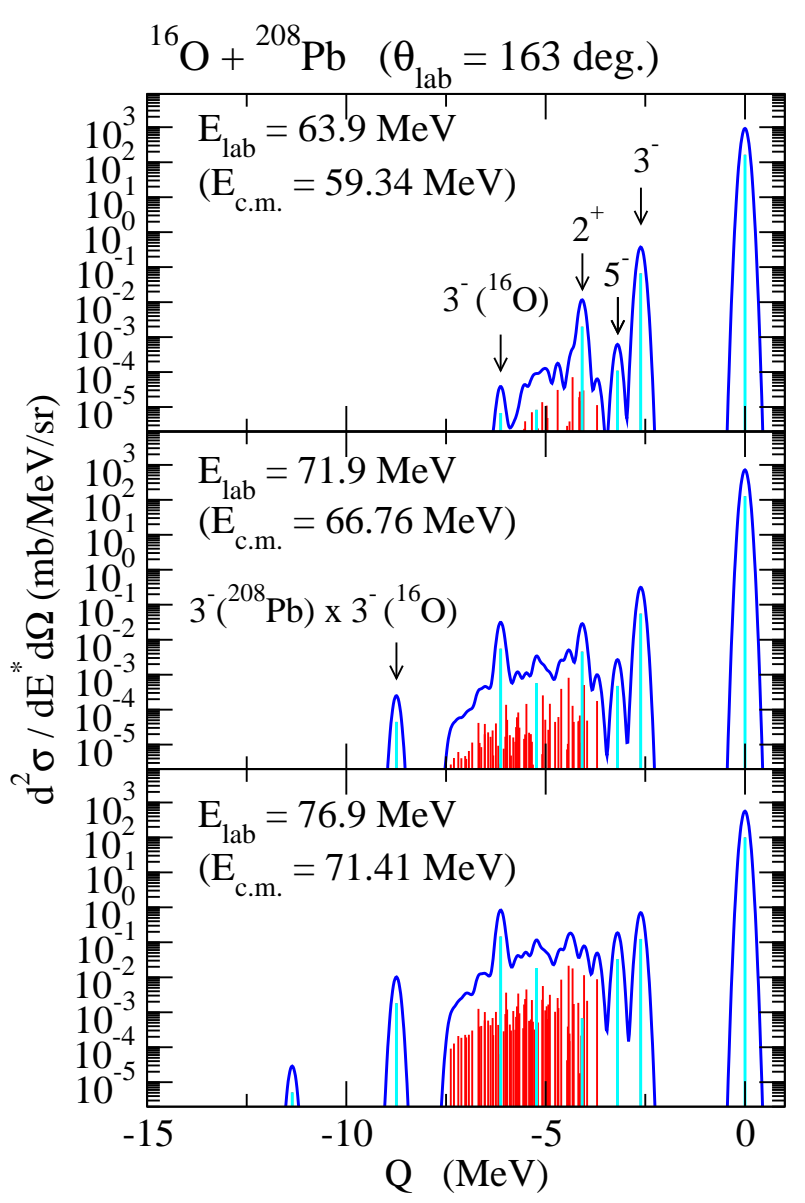

Fig. 3. The $Q$-value spectra for the quasi-elastic ${ }^{16} \mathrm{O}+{ }^{208} \mathrm{~Pb}$ scattering at $\theta_{\mathrm{lab}}=163^{\circ}$ for three different incident energies as shown in the figure. For the presentation purpose, the discrete spectra are smeared with a Gaussian function.

excitation energies and those high-lying states in general lead to the potential renormalization [4]. Actually, by shifting the thin solid lines in energy, these match well with the thick solid line (see the dashed line). Therefore, the non-collective excitations in the isolated nuclei do not alter much the energy dependence of the fusion excitation function, and are not responsible for the deep subbarrier hindrance, as one can see also in the logarithmic derivative shown in the bottom panel. In order to account for the deep subbarrier hindrance, the dynamical process considered in Ref. [21] should be taken into account.

Although the effect is not large for this system, the non-collective excitations slightly modify the shape of the barrier distribution as shown in the third panel of Fig. 1. That is, the bump at $E \sim 77 \mathrm{MeV}$ is somewhat smeared by the non-collective couplings. This is consistent with the experimental observation for the ${ }^{20} \mathrm{Ne}+{ }^{90,92} \mathrm{Zr}$ systems [5] as well as with the result of a one-dimensional schematic model [6].

Figure 2 shows the cross sections for the corresponding quasi-elastic scattering at the scattering angle of $\theta_{\text {lab }}=$

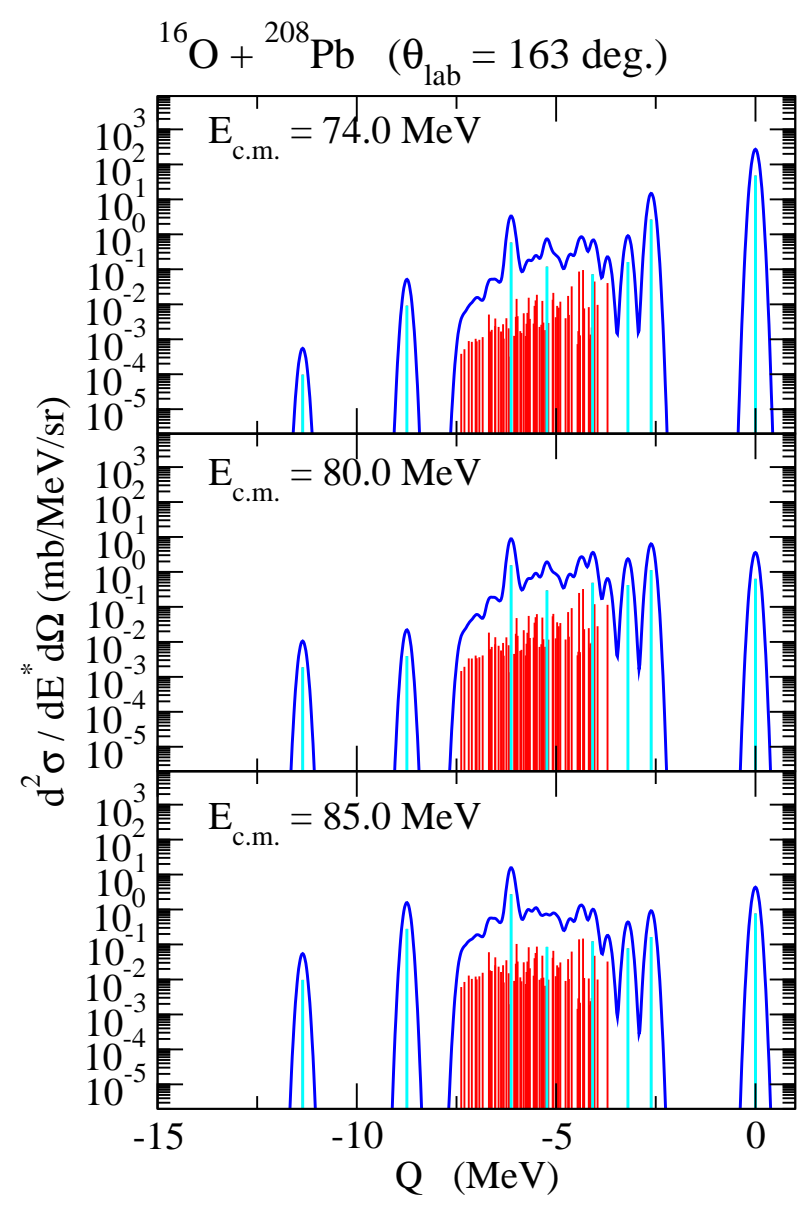

Fig. 4. Same as Fig. 3, but for other three different incident energies.

$170^{\circ}$ and the quasi-elastic barrier distribution[11,19],

$$
D_{\mathrm{qel}}=-\frac{d}{d E}\left(\frac{d \sigma_{\mathrm{qel}}}{d \sigma_{R}}\right),
$$

where $\sigma_{R}$ is the Rutherford cross section. The effects of the non-collective excitations are similar to those in the fusion reaction. That is, the non-collective excitations shift the excitation function in energy and at the same time somewhat smear the barrier distribution. Notice, however, that the degree of smearing seems larger in the quasi-elastic barrier distribution as compared to the fusion barrier distribution.

Figures 3 and 4 show the $Q$-value spectra for quasielastic scattering calculated at $\theta_{\mathrm{lab}}=163^{\circ}$ at six different incident energies. For a presentation purpose, we smear the discrete spectra with a Gaussian function,

$$
\bar{f}(E)=\sum_{i} f_{i} \cdot \frac{1}{\sqrt{\pi} \sigma} e^{-\left(E-E_{i}\right)^{2} / \sigma^{2}},
$$

with $\sigma=0.1 \mathrm{MeV}$. At the lowest energy shown in the figure, that is, $E_{\text {c.m. }}=59.34 \mathrm{MeV}$, only the elastic peak $(Q=0)$ and the peak corresponding to the $3^{-}$state in ${ }^{208} \mathrm{~Pb}$ are important. As energy increases, the non-collective excitations gradually become significant. This feature agrees with the experimental $Q$-value distribution for the same 
system shown in Refs. $[12,13]$, although the transfer compoents have to be subtracted from the experimental data in order to compare directly with the present results.

\section{Summary}

We have solved the coupled-channels equations for the ${ }^{16} \mathrm{O}$ $+{ }^{208} \mathrm{~Pb}$ reaction by including the non-collective excitations in the ${ }^{208} \mathrm{~Pb}$ nucleus. We have shown that the energy dependence of the fusion and quasi-elastic excitation functions is not modified much by the non-collective couplings, while the structure of the barrier distributions is somewhat smeared due to the non-collective excitations. The noncollective couplings thus provide a promising way to explain the observed difference in the quasi-elastic barrier distribution for the ${ }^{20} \mathrm{Ne}+{ }^{90,92} \mathrm{Zr}$ systems [5]. In the $Q$ value distribution, we found that the contribution from the non-collective excitations increases significantly as the incident energy increases. This feature is in accordance with the experimental data shown in Refs. $[12,13]$.

This work was supported by the Grant-in-Aid for Scientific Research (C), Contract No. 22540262 from the Japan Society for the Promotion of Science.

\section{References}

1. K. Hagino, N. Rowley, and A.T. Kruppa, Comp. Phys. Comm. 123, 143 (1999).

2. M. Dasgupta, D.J. Hinde, N. Rowley, and A.M. Stefanini, Annu. Rev. Nucl. Part. Sci. 48 (1998) 401.

3. A.B. Balantekin and N. Takigawa, Rev. Mod. Phys. 70 (1998) 77.

4. N. Takigawa, K. Hagino, M. Abe, and A.B. Balantekin, Phys. Rev. C49 (1994) 2630.

5. E. Piasecki et al., Phys. Rev. C80 (2009) 054613.

6. S. Yusa, K. Hagino, and N. Rowley, Phys. Rev. C82 (2010) 024606.

7. J.O. Newton et al., Phys. Rev. C70 (2004) 024605.

8. C.L. Jiang et al., Phys. Rev. Lett. 89 (2002) 052701; Phys. Rev. C79 (2009) 044601, and references therein.

9. M. Dasgupta et al., Phys. Rev. Lett. 99 (2007) 192701. 10. A.M. Stefanini et al., Phys. Rev. C78 (2008) 044607; Phys. Lett. B679 (2009) 95.

11. H. Timmers et al., Nucl. Phys. A584 (2005) 190.

12. M. Evers et al., Phys. Rev. C78 (2008) 034614

13. C.J. Lin et al., Phys. Rev. C79 (2009) 064603.

14. W.T. Wagner, G.M. Crawley, G.R. Hammerstein, and H. McManus, Phys. Rev. C12 (1975) 757.

15. M.B. Lewis, F.E. Bertrand, and C.B. Fulmer, Phys. Rev. C7 (1973) 1966

16. G.S. Adams et al., Phys. Lett. 91B (1980) 23

17. A. Scott, N.P. Mathur, and F. Petrovich, Nucl. Phys. A285 (1977) 222.

18. P. Demetriou, A. Marcinkowski, and P.E. Hodgson, Nucl. Phys. A596 (1966) 67.

19. K. Hagino and N. Rowley, Phys. Rev. C69 (2004) 054610 .
20. R.A. Broglia and A. Winther, Heavy Ion Reactions, (Addison-Wesley, Redwood City, CA, 1991).

21. T. Ichikawa, K. Hagino, and A. Iwamoto, Phys. Rev. Lett. 103 (2009) 202701; Phys. Rev. C75 (2007) 064612; Phys. Rev. C75 (2007) 057603.

22. C.R. Morton et al., Phys. Rev. C60 (1999) 044608.

23. M. Dasgupta et al., Phys. Rev. Lett. 99 (2007) 192701.

24. H. Timmers, Ph. D. thesis, Australian National University, 1996. 